\title{
ELECTRIC AND MAGNETIC FIELDS DUE TO DIRAC PARTICLES IN FRW SPACETIME
}

\author{
P.R. Dhungel*, S. K. Sharma** and U. Khanal*
}

*Central Department of Physics, Tribhuvan University, Kirtipur, Nepal.

**B.P. Koirala Memoriala Planetarium, Observataroy and Science Museum Development Board, Singha Dubar, Kathmandu, Nepal.

\begin{abstract}
Some solutions of the Maxwell equations with Dirac particles for the source in FRW spacetime are discussed. The Green's function of the equation for the radial component of the Maxwell fields, $F_{r m}$ and $F_{\theta \varphi}$ is solved. Green's function is found to reduce to that of Minkowskian spacetime in the appropriate limit. Also, the Lienard-Wiechert type solution is derived. Also, the solutions with the Dirac particle current is also presented. It is found that the $\mathrm{F}_{\mathrm{r} m}$ is composed of even angular momentum states while the odd states constitute $\mathrm{F}_{\theta \varphi^{\circ}}$
\end{abstract}

Keywords: Dirac equation; NP formalism; FRW space-time; Maxwell field.

\section{INTRODUCTION}

In Newmann-Penrose formalism [1], the six components of the Maxwell field tensors are represented by three complex scalars $\varphi_{0}, \varphi_{1}$ and $\varphi_{2}$. In the tetrad frame we have chosen, $[2,3]$ they are

$$
\begin{gathered}
\phi_{0}=F_{\mu \nu} l^{\mu} m^{\nu}=\frac{1}{\sqrt{2} a^{3} \sin r}\left[F_{r \theta}+F_{\eta \theta}+\frac{i}{\sin \theta}\left(F_{\eta \theta}+F_{r \phi}\right)\right] \\
\phi_{1}=\frac{1}{2} F_{\mu \nu}\left(l^{\mu} n^{\nu}+\bar{m}^{\mu} m \nu\right)=\frac{1}{2 a^{2} \sin ^{2} r}\left[\sin ^{2} r F_{r \eta}+\frac{i}{\sin \theta} F_{\theta \phi}\right]
\end{gathered}
$$

and

$$
\phi_{2}=F_{\mu \nu} \bar{m}^{\mu} n^{\nu}=\frac{1}{2 \sqrt{2} a \sin r}\left[F_{r \theta}-F_{\eta \theta}+\frac{i}{\sin \theta}\left(F_{\eta \theta}-F_{r \phi}\right)\right]
$$

In projections of quantities in the tetrad frame, the number of $m$ minus the number of is called the spin weight, while the number of $l$ minus the number of $n$ is the boost weight. As a consequence, we see that $\varphi_{1}$ has spin and boost weight equal to zero and thus behaves as a spin zero (scalar) field. Similarly, $\varphi_{0}$ and $\varphi_{2}$ are spin +1 and -1 fields.

Next, the Maxwell equations, with source by the Dirac current (3) take on the form

$$
\begin{aligned}
& \sin r \mathcal{D}_{0}^{-} \Phi_{0}-\mathcal{L}_{1}^{+} \Phi_{1}=\frac{\sin r}{\sqrt{2}}\left|\Phi_{1 / 2}\right|^{2}\left(\left|Y_{1 / 2}\right|^{2}+\left|Y_{-1 / 2}\right|^{2}\right)=J_{0}^{+} \\
& \sin r \mathcal{D}_{-1}^{+} \Phi_{1}+\mathcal{L}_{0}^{-} \Phi_{0}=\frac{\sin r}{\sqrt{2}}\left(\Phi_{1 / 2}^{*} \Phi_{-1 / 2}-\Phi_{1 / 2} \Phi_{-1 / 2}^{*}\right) Y_{1 / 2} Y_{-1 / 2}^{*}=J_{1} \\
& \sin r \mathcal{D}_{-1}^{-} \Phi_{-1}+\mathcal{L}_{0}^{+} \Phi_{0}=J_{1}^{*}=-J_{-1}
\end{aligned}
$$

and

$$
\sin r \mathcal{D}_{0}^{+} \Phi_{0}-\mathcal{L}_{1}^{-} \Phi_{-1}=\frac{\sin r}{\sqrt{2}}\left|\Phi_{-1 / 2}\right|^{2}\left(\left|Y_{1 / 2}\right|^{2}+\left|Y_{-1 / 2}\right|^{2}\right)=J_{0}^{-}
$$

where we have substituted

$$
\Phi_{0}=\sqrt{2} a^{2} \sin ^{2} r \Phi_{1}, \Phi_{+1}=a^{3} \sin ^{2} r \Phi_{0}
$$

and

$$
\Phi_{-1}=2 a \sin ^{2} r \Phi_{2}
$$

The radial-temporal operator

$$
\mathcal{D}_{\mathrm{s}}^{ \pm}=\left(\frac{\partial}{\partial \theta} \mp \frac{i}{\sin \theta} \frac{\partial}{\partial \phi}+s \cot r\right)
$$

and the angular operator

$\mathcal{L}_{\mathrm{s}}^{ \pm}=\left(\frac{\partial}{\partial \theta} \mp \frac{i}{\sin \theta} \frac{\partial}{\partial \phi}+s \cot \theta\right)$, where $\eta$ is

the conformal time $\mathrm{dt}=\mathrm{a} \mathrm{d} \eta$ with $a$ equal to the scale factor

We can easily decouple the Maxwell equations to read [4]

$$
\left[\sin r \mathcal{D}_{-1}^{ \pm} \sin r D_{0}^{\mp}+\mathcal{L}_{1}^{ \pm} \mathcal{L}_{0}^{\mp}\right] \Phi_{0}=\sin r \mathcal{D}_{-1}^{ \pm} J_{0}^{ \pm}+\mathcal{L}_{1}^{ \pm} J_{ \pm 1}=S_{0}
$$

and

$$
\left[\sin r \mathcal{D}_{0}^{\mp} \sin r D_{-1}^{ \pm}+\mathcal{L}_{0}^{\mp} \mathcal{L}_{1}^{ \pm}\right] \Phi_{1}= \pm \sin r \mathcal{D}_{0}^{\mp} J_{ \pm 1} \mp \mathcal{L}_{0}^{\mp} J_{0}^{ \pm}=S_{ \pm 1}
$$

In Eq. (9), it is seen that $J_{0}$ is the source for the radial electric field and $\mathrm{J}_{1}$ for the radial magnetic field. The eigenfunctions of the angular operator are the well known spin-weighted spherical harmonics satisfying

$$
\mathcal{L}_{-(s-1)}^{\mp} \mathcal{L}_{\mathrm{s}}^{ \pm} Y_{ \pm}=-(l+s)(l-s+1) Y_{ \pm} ;
$$

we have identified these functions as the spherical harmonics formed with the Jacobi polynomials viz. 
$Y_{\theta s}={ }_{s} Y_{l}^{m}=\frac{e^{i m \phi}}{\sqrt{2 \pi}} N(1-\cos \theta)^{\frac{m+s}{2}}(1+\cos \theta)^{\frac{m-s}{2}} P_{l-m}^{(m+s, m-s)}(\cos \theta)$

while $\mathrm{Y}_{0}^{\mathrm{s}}=\mathrm{Y}_{l}^{m}$ are usual spherical harmonics. These are normalized. So,

$\int_{s} Y_{l_{1}{ }_{s}}^{m_{1}^{*}} Y_{l_{2}}^{m_{2}} d \Omega=\delta_{l_{1}, l_{2}} \delta_{m_{1}, m_{2}}$

are complete in

$\sum_{l, m} Y_{l}^{m^{*}}\left(\Omega^{\prime}\right)_{s} Y_{l}^{m}(\Omega)=$

$\delta^{2}\left(\Omega-\Omega^{\prime}\right),{ }_{s} Y_{l}^{m^{*}}=(-1)^{s+m}{ }_{-s} Y_{l}^{m}$

and satisfy the spin lowering operation

$$
\mathcal{L}_{\mathrm{s}}^{ \pm} Y_{ \pm s}= \pm \sqrt{(l+s)(l-s+1)} Y_{ \pm(s-1)}
$$

\section{Green's Function Of The Scalar Equation}

The Green's function of the scalar equation can be determined by working out the solution of the Eq. (8) with point source:

$$
\begin{aligned}
& \sin ^{2} r\left[\frac{\partial^{2}}{\partial r^{2}}-\frac{\partial^{2}}{\partial \eta^{2}}+\frac{1}{\sin ^{2} r} \mathcal{L}_{1}^{+} \mathcal{L}_{0}^{-}\right] G_{0}(\eta, r, \theta, \phi) \\
= & \delta\left(r-r^{\prime}\right) \delta\left(\eta-\eta^{\prime}\right) \delta\left(\theta-\theta^{\prime}\right) \delta\left(\phi-\phi^{\prime}\right)
\end{aligned}
$$

By standard technique, we can make an eigenfunction expansion of the Green's function[5] as

$$
\begin{gathered}
G\left(\eta, r, \theta, \phi: \eta^{\prime}, r^{\prime}, \theta^{\prime}, \phi^{\prime}\right)= \\
\frac{1}{2 \pi} \int_{-\infty}^{\infty} d \omega \sum_{k l m} \frac{e^{-i \omega\left(\eta-\eta^{\prime}\right)}}{\omega^{2}-k^{2}} \frac{R_{k}\left(r^{\prime}\right) R_{k}(r)}{\sin r \sin r^{\prime}} Y_{l}^{m^{*}}\left(\Omega^{\prime}\right) Y_{l}^{m}(\Omega)
\end{gathered}
$$

where the radial eighenfunctions are the normalized, appropriately weighted Gegenbauer polynomials[6].

$$
R_{k}=N(\sin r)^{l+1} C_{k-l-1}^{l+1}(\cos r)
$$

The addition theorem of spherical harmonics gives

$\sum_{m}{ }_{l} Y_{m}^{*}\left(\Omega^{\prime}\right){ }_{l} Y_{m}(\Omega)=\frac{z l+1}{4 \pi} C_{l}^{1 / 2}(\cos \beta)$

with

$$
\cos \beta=\cos \theta \cos \theta^{\prime}+\sin \theta \sin \theta^{\prime} \cos \left(\phi-\phi^{\prime}\right)
$$

The $\omega$ integral can be done by the method of residues to give

$$
\frac{1}{2 \pi} \int_{-\infty}^{\infty} d w \frac{e^{-i w\left(\eta-\eta^{\prime}\right)}}{w^{2}-k^{2}}=-\frac{\sin k\left(\eta-\eta^{\prime}\right)}{k}
$$

with these results we can rewrite the summation as

$$
\begin{aligned}
& G_{0}=-\frac{1}{4 \pi} \sum_{k=0}^{\infty} \sum_{l=0}^{k} \frac{\sin \left[(k+1)\left(\eta-\eta^{\prime}\right)\right]}{(k+1)}\left(\sin r \sin r^{\prime}\right)^{l} \\
& C_{k-l}^{l+1}\left(\cos r^{\prime}\right) C_{k-l}^{l+1}(\cos r) C_{l}^{\frac{1}{2}}(\cos \rho) \\
& =-\frac{1}{4 \pi} \sum_{k=0}^{\infty} \sin \left[(k+1)\left(\eta-\eta^{\prime}\right)\right] C_{k}^{(1)}(\cos \rho)
\end{aligned}
$$

where we have used the addition theorem of Gegenbauer polynomial from reference [7] and $\cos \rho=\cos r \cos r '+$ $\sin r \sin r^{\prime} \cos \beta$.

Now

$$
C_{k}^{1}(\cos \rho) \quad=\quad \frac{\sin [(k+1) \rho]}{\sin \rho}
$$

So, we find

$$
G_{0}=-\frac{1}{4 \pi \sin \rho} \quad\left[\delta\left(\eta-\eta^{\prime}-\rho\right)-\delta\left(\eta-\eta^{\prime}+\rho\right)\right]
$$

representing the retarded and advanced Green's function. Thus, the solution for the retarded scalar field is

$$
\Phi_{0}(\eta, r, \theta, \phi)=-\frac{1}{4 \pi} \int_{0}^{\pi} \frac{d r^{\prime}}{\sin \rho} \int d \Omega^{\prime} S_{0}\left(\eta-\rho, r^{\prime}, \Omega^{\prime}\right)
$$

We can even solve for Lienard-Wiechert like field in closed FRW space time for a point source moving

$\vec{\xi}(\eta)$ given by $\mathrm{S}_{0}=\delta\left[\overrightarrow{r^{\prime}}-\vec{\xi}\left(\eta^{\prime}\right)\right]$ to find

$$
\Phi_{0}(\eta, r, \theta, \phi)=-\left.\frac{1}{4 \pi} \frac{1}{\sin \left[\rho\left(\eta^{\prime}\right)\right]} \frac{1}{\left[1+\frac{d \rho\left(\eta^{\prime}\right)}{d \eta}\right]}\right|_{\eta^{\prime}=\eta-\rho\left(\eta^{\prime}\right)}
$$

In the limit to flat FRW space time given by $\mathrm{r}<<1, \xi$ $<<1$ and $\sin \rho \sim \rho$, and

$$
\begin{aligned}
& \cos \rho \rightarrow 1-\frac{\rho^{2}}{2} \\
& \approx\left(1-\frac{r^{2}}{2}\right)\left(1-\frac{\xi^{2}}{2}\right)+r \xi \cos \beta \\
& =1-\frac{1}{2}|\vec{r}-\vec{\xi}|^{2} \text { gives } \\
& \Phi_{0} \rightarrow-\frac{-}{4 \pi} \frac{\vec{r}}{\left|\vec{r}-\vec{\xi}\left(\eta^{\prime}\right)\right|-\left.\frac{d \vec{\xi}}{d \eta} \cdot\left(\vec{r}-\vec{\xi}\left(\eta^{\prime}\right)\right)\right|_{\eta^{\prime}=\eta-\left|\vec{r}-\vec{\xi}\left(\eta^{\prime}\right)\right|}}
\end{aligned}
$$

which is the familiar Lienard-Wiechert solution.

\section{Radial Maxwell fields due to Dirac source}

In this regard, let us solve for the field in the very early universe when the Dirac particles are copiously produced so that all the available states are fully occupied. When

$$
\mathcal{L}_{0}^{ \pm} Y_{ \pm \frac{1}{2}} Y_{\mp \frac{1}{2}}^{*}=-\left(l+\frac{1}{2}\right)\left(\left|Y_{1 / 2}\right|^{2}-\left|Y_{-1 / 2}\right|^{2}\right)
$$

we sum the source on the left of Eq. (8), the second term containing will give zero. The consequence is that there is no source for the radial magnetic field. This 
is general in that whenever the $\pm \mathrm{m}$ states are paired, their contributions cancel out. In this case, there is an isotropic source of electric field only as .

$$
\sum_{m}\left(\left|Y_{1 / 2}\right|^{2}+\left|Y_{-1 / 2}\right|^{2}\right)=\frac{2(2 l+1)}{4 \pi}
$$

Thus the Maxwell Eq. (9) reduces to

$$
\begin{aligned}
& \mathcal{D}_{0}^{\mp}\left[\sin ^{2} r F_{r \eta}\right]=\sum_{k l}\left|\phi_{ \pm \frac{1}{2}}\right|^{2} \frac{2(2 l+1)}{4 \pi} \\
& \therefore . \\
& \frac{\partial}{\partial r} \sin ^{2} r F_{r \eta}=\sum_{k l} \frac{(2 l+1)}{4 \pi}\left(\left|\phi_{\frac{1}{2}}\right|^{2}+\left|\phi_{-\frac{1}{2}}\right|^{2}\right)
\end{aligned}
$$

and

$$
\frac{\partial}{\partial r} \sin ^{2} r F_{r \eta}=\sum_{k l} \frac{(2 l+1)}{4 \pi}\left(\left|\phi_{\frac{1}{2}}\right|^{2}-\left|\phi_{-\frac{1}{2}}\right|^{2}\right)
$$

The solution is

$$
\begin{aligned}
& 4 \pi \sin ^{2} r F_{r \eta}=\frac{1}{2} \sum_{k l}(2 l+1) \int_{0}^{r} d r^{\prime}\left(\left|Z_{+}\right|^{2}+\left|Z_{-}\right|^{2}\right) \\
&=\sum_{k=0 l=0}^{\infty} \sum_{0}^{k} \int_{0}^{r} d r \sin r(1-\cos r)^{l+\frac{1}{2}}(1+\cos r)^{l-\frac{1}{2}} \\
& {\left[\left\{P_{k-l}^{\left(l+\frac{1}{2}, l+\frac{1}{2}\right)}(\cos r)\right\}^{2}\left|T_{+}\right|^{2}+\left\{P_{k-l}^{\left(l+\frac{1}{2}, l+\frac{1}{2}\right)}(\cos r)\right\}^{2}\left|T_{-}\right|^{2}\right] }
\end{aligned}
$$

where are appropriately weighted and normalized Jacobi polynomials, is just a statement of Gauss law, as the right handside is the co-moving number of enclosed particles. Here to first order WKB,

$$
\left|T_{ \pm}\right|^{2}=1 \pm \frac{a M}{\sqrt{\left(k+\frac{3}{2}\right)^{2}+a^{2} M^{2}}}
$$

The field intensity $\mathrm{F}_{\mathrm{r}}$ are shown in Fig (1) for different values of $\mathrm{M}$ and in Fig. (2) for some values of the comoving momentum $\mathrm{k}$ of the Dirac particles. We take those values to be representative of the proton and electron. At any finite time after the big bang, we see that the protons produce a stronger field than the electron. The two fields do not have the same magnitude even for the lowest state $\mathrm{k}=0$, and the heavier mass consistently produces stronger field of higher momentum also. These are exact solutions of the field equations, and we feel that this effect will have strong consequence on the formation of hydrogen and other atoms.

Next, we discuss the solutions in the case after particleantiparticle annihilation when the electron number becomes drastically reduced. Then there will not always be pairing of the $\pm \mathrm{m}$ states. For this, we can use the well known generalized Clebsch Gordon expansion

$$
\begin{aligned}
& s_{1} Y_{l_{1}}^{m_{1} s_{2}} Y_{l_{2}}^{m_{2}}= \\
& \sum_{l=\left|l_{1}-l_{2}\right|}^{l_{1}+l_{2}} \sqrt{\frac{\left(2 l_{1}+1\right)\left(2 l_{2}+1\right)(2 l+1)}{4 \pi}}\left(\begin{array}{ccc}
l_{1} & l_{2} & l \\
m_{1} & m_{2} & -\left(m_{1}+m_{2}\right)
\end{array}\right) \\
& \left(\begin{array}{ccc}
l_{1} & l_{2} & l \\
-s_{1} & -s_{2} & s_{1}+s_{2}
\end{array}\right){ }_{s_{1}+s_{2}} Y_{l}^{m_{1}+m_{2}}
\end{aligned}
$$

where $\left(\begin{array}{ccc}j_{1} & j_{2} & j \\ m_{1} & m_{2} & m\end{array}\right)$ are the wigner 3-j symbol. Hence, we find

$\left|Y_{+}\right|^{2}+\left|Y_{-}\right|^{2}=2(-1)^{m-\frac{1}{2}} \sum_{l=0}^{l-\frac{1}{2}} \sqrt{\frac{(2 l+1)^{2}(4 l+1)}{4 \pi}}$

$\left(\begin{array}{ccc}l & l & 2 L \\ m & -m & 0\end{array}\right)\left(\begin{array}{ccc}l & l & 2 L \\ \frac{1}{2} & -\frac{1}{2} & 0\end{array}\right){ }_{0} Y_{2 L}^{0}$

contributes only the even angular momentum states. Similarly,

$\mathcal{L}_{1}^{ \pm} Y_{ \pm \frac{1}{2}} Y_{\mp \frac{1}{2}}^{*}=$

$2\left(l+\frac{1}{2}\right)(-1)^{m-\frac{1}{2}} \sum_{l=0}^{l-\frac{1}{2}} \sqrt{\frac{(2 l+1)^{2}(4 l+3)}{4 \pi}}$

$\left(\begin{array}{ccc}l & l & 2 L+1 \\ m & -m & 0\end{array}\right)\left(\begin{array}{ccc}l & l & 2 L+1 \\ \frac{1}{2} & -\frac{1}{2} & 0\end{array}\right){ }_{0} Y_{2 L}^{0}$

contributes the odd states. So, we can conclude that the radial electric field is composed of even angular momentum states and the odd ones compose radial magnetic field. These solutions and the solutions for $\varphi_{ \pm 1}$ will be considered in future work.

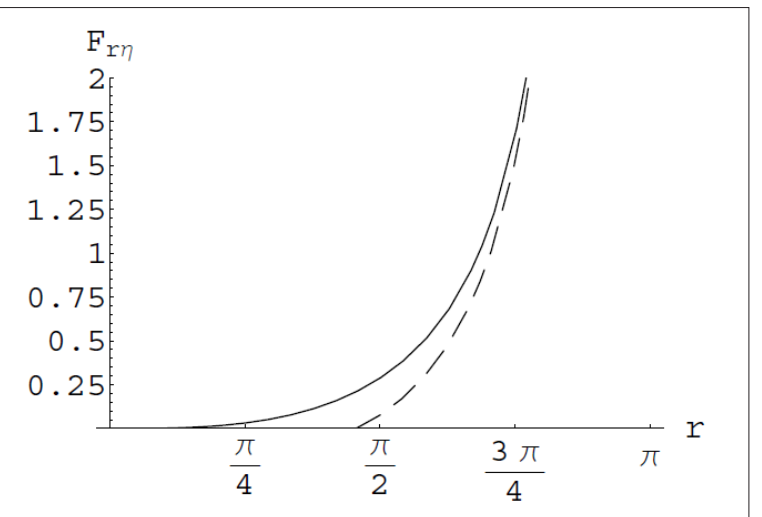

Figure 1: The radial dependence of field intensity $F_{r \eta}$ for two different masses. Solid and dashed lines are for $M=100$ and 0.01 respectively.

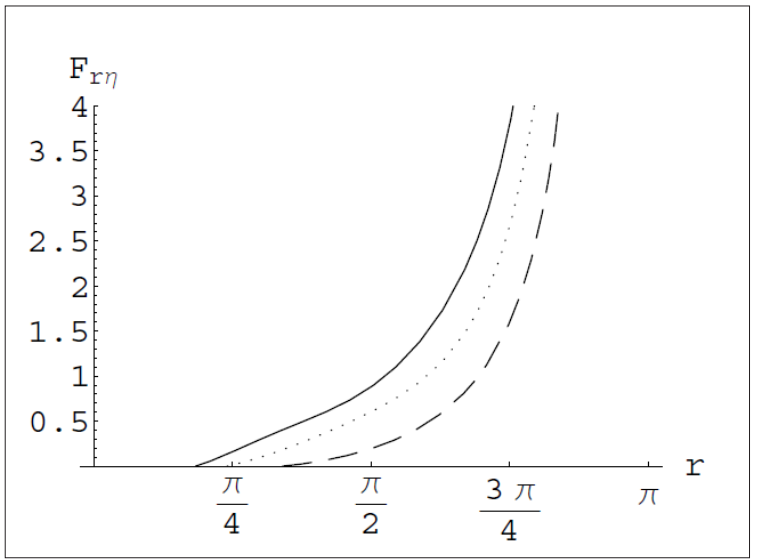

Figure 2: The radial dependance of field intensity $F_{r \eta}$ for different values of comoving momentum $k$. The dashed, dotted and solid lines are for $\mathrm{k}=\mathbf{0}, 1$ and 2 respectively. 


\section{CONCLUSION}

We have solved some of the Maxwell equations in FRW spacetime with the source given by Dirac field. The variables are all separable. For the scalar field representing the components $F_{r n}$ and $F_{\theta \varphi}$, we are able to solve the Green's function which reduces to the familiar ones of electrodynamics in the appropriate limit to flat case. The retarded part is used to derive the Lienard Wiechert type solution for moving point source.

In section 3, we have considered the source given by Dirac potentials. In the case that all the available states of the Dirac particle are filled, the magnetic field $F_{\theta \varphi}$ is found to vanish. When we compare the electric filed strength $\mathrm{F}_{\mathrm{r} \eta}$ due to Dirac particles of mass ratio 10000 , they are somewhat different with the lighter one contributing more. At this lowest level the fields due to proton and electron are not exactly opposite of each other. This should have observable effects on the formation of atoms and particularly on the time of decoupling. When we consider individual azimuthal states of Dirac particles, the source term vanishes. When we consider Dirac particles after the particle-antiparticle annihilation, the number density is greatly decreased so that all the available states cannot be occupied. In particular, if all the $\pm \mathrm{m}$ azimuthal states are not paired, then the source for $\mathrm{F}_{\mathrm{r}}$ and $\mathrm{F}_{\theta \varphi}$ do not vanish. In that case $\mathrm{F}_{\mathrm{r}}$ is composed of even angular momentum $(l)$ states and $F_{\theta \varphi}$ is composed of odd states. So, there is the possibility of the existence of a primordial magnetic field generated just after $\mathrm{e}^{+}$- $\mathrm{e}^{-}$annihilation.

\section{REFERENCES}

[1] Newman, E. T. and Penrose, R. J. 1962. Math. Phys. 3: 566.

[2] Chandrasekhar, S. 1983. The Mathematical Theory of Black Holes. (Clarendon Press, Oxford).

[3] Khanal, U. 2006. Class. Quantum. Grav. 23: 4353.

[4] Dhungel, P.R. and Khanal, U. 2013. Chinese Journal of Physics 51, 882, arXiv:1109.6412v2 [astro-ph.CO].

[5] Mathews, J. and Walker, R. L. 1970. Mathematical Methods of Physics, Benjamin, Menlo Park.

[6] Chandrasekhar, S. 1983. The Mathematical Theory of Black Holes, (Clarendon Press, Oxford).

[7] Abramowitz, M. and Stegun, I. A. 1976. Handbook of Mathematical Functions, New York, Dover.

[8] Gradshteyn, I. S. and Ryzhik, I. M. 1980. Table of Integral, Series and Products, (Academic Press, New York). 